\title{
Quand les Celtes deviennent orthodoxes
}

De l'exaltation du passé à la modernité religieuse

Katerina Seraïdari et Alexis Léonard

\section{CpenEdition}

Journals

Édition électronique

URL : http://journals.openedition.org/assr/8933

DOI : 10.4000/assr.8933

ISSN : $1777-5825$

Éditeur

Éditions de l'EHESS

Édition imprimée

Date de publication : 1 septembre 2007

Pagination : 79-99

ISBN : 978-2-7132-2144-6

ISSN : 0335-5985

Référence électronique

Katerina Seraïdari et Alexis Léonard, "Quand les Celtes deviennent orthodoxes », Archives de sciences sociales des religions [En ligne], 139 | juillet - sepembre 2007, mis en ligne le 19 novembre 2010,

consulté le 19 avril 2019. URL : http://journals.openedition.org/assr/8933 ; DOI : 10.4000/assr.8933

Ce document a été généré automatiquement le 19 avril 2019

(c) Archives de sciences sociales des religions 


\title{
Quand les Celtes deviennent orthodoxes
}

\author{
De l'exaltation du passé à la modernité religieuse
}

Katerina Seraïdari et Alexis Léonard

1 Ainsi qu'en témoignent de nombreux ouvrages et expositions récents, les Celtes sont présentés comme les ancêtres de l'Europe et certains auteurs n'hésitent pas à dire que l'Union Européenne ne fait que reproduire ce que les Celtes avaient créé il y a 2500 ans (Sims-Williams, 1998). Six « pays » constituent la Celtie traditionnelle : l'Irlande, l'Écosse, le Pays de Galles, les Cornouailles, l'île de Man et la Bretagne. La présence sur la plus grande partie du continent européen de populations celtes pendant l'Antiquité a permis à des pays comme la Suisse ou la France de revendiquer eux aussi des racines celtiques. Par ailleurs, l'émigration massive qu'ont connue des pays comme l'Irlande, notamment vers les États-Unis où quelque quarante millions d'Américains se réclament d'origine irlandaise, a eu pour résultat d'élargir la zone d'influence des Celtes en dehors de l'Europe. La Celtie semble donc être un territoire à géométrie variable que l'on manipule à volonté, au point de l'identifier parfois à l'Occident.

2 Dans le cadre d'une spiritualité celtique très en vogue, on peut actuellement observer deux tendances: des mouvements néo-druidiques, mais aussi une articulation entre ces discours identitaires et les trois branches du christianisme. Nous analyserons ici la rencontre opérée entre celtisme et orthodoxie, qui paraît d'autant plus surprenante qu'aucun pays orthodoxe n'associe son histoire à celle des Celtes, et que le christianisme orthodoxe est traditionnellement attaché à l'Orient. Comme nous le verrons, l'héritage orthodoxe est remanié afin de s'accorder aux expériences de populations qui ont connu soit le catholicisme soit le protestantisme.

3 Assimiler l'Église orthodoxe à une "famille » d'Églises organisées selon des critères géographiques et non ethniques constitue une qualité, depuis longtemps exaltée par les orthodoxes, qui devient chez ceux que l'on pourrait appeler les «Celtes orthodoxes » un argument majeur. Elle permet de concilier le besoin d'afficher une spécificité locale, nationale ou supranationale (dans le cas de la Celtie) et le désir de s'apparenter à une structure religieuse d'envergure. Néanmoins, il s'agit d'une image idéalisée qui entraîne 
nécessairement des contradictions. Ainsi, on ne manque pas de souligner que les Églises orthodoxes «traditionnelles » se sont enfermées dans des "ghettos ethniques » et ont développé des discours identitaires exclusifs. On oscille en permanence entre l'affirmation d'un esprit égalitaire et décentralisé qui caractériserait les relations entre ces Églises locales ou nationales, et une réalité qui impose ses clivages et ses propres principes de hiérarchisation.

4 L'orthodoxie étant la branche du christianisme la moins étudiée jusqu'à aujourd'hui, il n'est pas surprenant que le renouveau religieux qui se rattache à elle constitue un sujet de recherche encore plus marginal. C'est cette lacune qui a motivé, en partie, notre démarche. Mais cette étude préliminaire est surtout la rencontre d'un terrain associant deux spécialités : l'orthodoxie et ses formes actuelles en Grèce pour Katerina Seraïdari et la sainteté médiévale en Irlande et les mouvements celtiques actuels pour Alexis Léonard.

5 Afin de mieux connaître ce mouvement religieux, nous avons eu recours à différentes sources : enquête de terrain et utilisation d'une bibliographie «atypique $»^{1}$, mais aussi recherches sur Internet. Nous montrerons comment ce mouvement religieux interprète, d'un côté, l'histoire du christianisme et, de l'autre, l'histoire européenne. Le but est de suivre la manière dont ce mouvement est né et sur quelles bases symboliques et historiques il a construit son identité et sa "mémoire généalogique ». Nous étudierons, plus particulièrement, l'Église orthodoxe celtique (EOC), dont le siège primatial se trouve au monastère breton de la Sainte-Présence ${ }^{2}$ à Saint-Dolay. Selon le préambule de son règlement intérieur, rédigé en $2002^{3}$, cette Église « est la résurgence de la vénérable Église Celtique, fondée par saint Joseph d'Arimathie en l'an 37 et saint Aristobule envoyé par l'apôtre Paul. Elle s'est développée dans toutes les Îles britanniques, la Bretagne par l'immigration des tribus bretonnes et le continent européen par ses nombreuses missions irlandaises, avant de perdre progressivement sa souveraineté jusqu'à la fin du douzième siècle ». La venue de Joseph d'Arimathie à Glastonbury et l'entrée de la Grande-Bretagne dans l'histoire chrétienne sont donc les deux événements majeurs sur lesquels cette Église s'appuie pour revendiquer autant un caractère apostolique qu'une spécificité celtique.

\section{Glastonbury, le lieu originel}

6 Situé au sud-ouest de l'Angleterre, à trente kilomètres de la mer, Glastonbury est identifié à l'île d'Avalon ${ }^{4}$ et au destin exceptionnel du roi Arthur. Vers 1135, Guillaume de Malmesbury écrit le De antiquitate Glastonie ecclesie : ces légendes élaborées et rassemblées par un érudit catholique continuent de nourrir le discours des "Celtes Orthodoxes ». La confusion entre mythe et histoire est permanente et trouve ses sources dans un imaginaire médiéval pris à la lettre. Ainsi que $\operatorname{Mgr}_{\operatorname{Marc}}^{5}(2000: 15)$ le met en exergue, « la légende est plus vraie que l'histoire » (phrase qu'il attribue à Socrate).

Une référence primordiale est le Saint Graal, la coupe utilisée par Jésus lors de la Cène et dans laquelle le sang du Christ aurait été recueilli. Il aurait été ensuite apporté en Angleterre par Joseph d'Arimathie, considéré comme l'apôtre de Grande-Bretagne, qui serait arrivé à Glastonbury accompagné de douze disciples et y aurait construit une église dédiée à la Vierge. Ainsi, par le biais de Glastonbury, la «naissance » et l'importance de l'Église britannique se trouvent liées, plus ou moins directement, à la personne du Christ ${ }^{6}$. 
8 Selon une autre version, Joseph d'Arimathie aurait été un marchand venu en Angleterre afin d'acheter l'étain nécessaire à la fabrication du bronze. Lors d'un de ses voyages, il aurait été accompagné par Jésus encore enfant ${ }^{7}$. Cette légende semble avoir inspiré William Blake (1757-1827), qui était lui-même un néo-druide: dans son poème «Jérusalem » (parfois appelé « Hymne de Glastonbury »), il se demande si Jésus n'a pas foulé le sol britannique. Afin de défendre ces traditions, Mgr Marc (2000 : 19-21) soutient qu'« un peuple d'origine orientale ou juive vivait en Cornouailles ou ailleurs dans les îles Britanniques ». Il fait aussi référence aux nombreux saints celtes qui portent des noms hébreux (comme David, Samson, Daniel...) et « aux similitudes étonnantes entre l'hymne de saint Patrick et les invocations hébraïques anciennes ».

9 Nous avons visité la ville de Glastonbury durant l'été 2000. De ce passé glorieux, il ne reste que les vestiges de l'ancienne abbaye. Dans cette ville de 9000 habitants, magasins et centres New Age abondent depuis les années 1970, ce qui montre bien la place que la " commercialisation de la spiritualité » tient dans l'économie locale. Le long de la rue centrale (High Street), on trouve une boutique appelée The Orthodox Way : ce magasin est tenu par un pope, John Ives, habillé à la manière orthodoxe (la seule différence étant une croix gaélique sur sa toque); il vend des objets de culte (cierges, encens, tenues de prêtre...), des icônes et des livres. À côté de la caisse, se trouve une photo du monastère breton (dont il sera question infra). Au cours de la conversation, il nous apprend qu'il était pasteur anglican avant de devenir orthodoxe, avec sa femme, en $1994^{8}$. Bien que sa " paroisse » ne rassemble que cinq convertis, l'orthodoxie est, selon lui, la troisième ou quatrième religion actuellement en Angleterre («bien avant les baptistes et les méthodistes ", ajoute-t-il) et la seule à gagner du terrain. Il précise que la messe est donnée en anglais, tandis que dans le monastère breton duquel il dépend, elle est donnée en breton; il exprime son admiration pour ce monastère. Par ailleurs, il revendique des racines celtiques et évoque une tradition familiale, transmise par son oncle, selon laquelle ses aïeux seraient des Bretons venus en Angleterre lors de l'invasion normande; même son nom de famille, Ives, serait d'origine celtique ${ }^{9}$.

10 À l'arrière de la boutique, au premier étage d'un bâtiment qui la jouxte, le pope nous conduit dans une longue pièce transformée en petite chapelle dédiée à Our Lady of Glastonbury; c'est ici que la messe est célébrée chaque samedi et dimanche. La décoration de cette chapelle n'a pas la richesse et l'éclat que l'on trouve généralement dans les églises orthodoxes : de simples reproductions d'icônes sur papier habillent les murs et l'iconostase. Dès qu'on entre dans la pièce, le pope ouvre le rideau qui cache l'autel (geste surprenant pour quelqu'un habitué à la tradition orthodoxe grecque...). L'autel est décoré d'une fresque monochrome représentant la Vierge avec le Christ sur ses genoux; elle est assise sur un trône de bois tressé rappelant, selon le pope, la légende de l'aubépine de Joseph d'Arimathie. Ce dernier aurait planté dans la terre de Glastonbury son bâton, qui se serait transformé en une aubépine (le Holy Thorn) donnant miraculeusement des fleurs chaque Noël. Selon Mgr Marc (2000: 8), « cet épineux appartient à une espèce qui pousse en Palestine et qui fleurit en hiver ». La botanique sert ainsi de preuve à la venue de Joseph d'Arimathie en Angleterre. Bien évidemment, cette tradition rappelle aussi la légende, selon laquelle saint Joseph fut choisi comme époux pour Marie parce que sa baguette avait fleurie. Dans ce cas, la confusion ne se produit pas seulement entre deux personnages homonymes, mais aussi entre leurs fonctions, puisque Joseph d'Arimathie est associé à la mort du Christ et le second à sa naissance. Mgr Marc (2000:28) écrit que « certaines statues de Joseph d'Arimathie ayant disparues, furent remplacées par saint 
Joseph, l'époux de la Vierge Marie ». Le rôle de ces récits est de renforcer, d'une part, l'importance de Joseph d'Arimathie (fondateur supposé de la petite chapelle Sainte-Marie de Glastonbury) dans la vie du Christ ; et d'autre part, la sainteté du lieu en augmentant le nombre des repères sacrés.

Dans un deuxième temps, il faut associer cet endroit emblématique (qui ne se trouve pas en fait sur une terre celtique, mais en Angleterre) aux régions celtiques, afin que la notion d'Église celtique et apostolique soit justifiée. Sur la fresque qui décore la chapelle orthodoxe de Glastonbury, Marie est entourée de deux saints: à sa gauche, Joseph d'Arimathie portant le Saint Graal, le suaire du Christ et le bâton fleuri, à sa droite, saint Aristobule. Ce saint, peu connu mais très ancien, est évoqué afin d'assurer le lien entre les débuts de l'Église britannique, les origines du christianisme et l'Orient. Il aurait été nommé évêque d'Angleterre par l'apôtre Paul ${ }^{10}$; il serait également l'un des soixantedouze disciples du Christ. La fresque, peinte par un haut membre du monastère celtique orthodoxe d'York, résume donc les éléments fondateurs de la «mythologie sacrée » de Glastonbury.

Sur le mur de gauche, on trouve l'icône de Our Lady of Glastonbury à laquelle la chapelle doit son nom. Le pope nous explique qu'une reproduction fut achetée en 1997 par un couple d'Américains orthodoxes, qui l'offrit à leur église à Atlanta. Quelque temps après, il reçut une lettre racontant que durant Noël 1997, cette copie avait exudé du parfum, à l'endroit où se trouve le brin fleuri d'aubépine (précisément entre les mains de la Vierge et les pieds du Christ). Que la copie d'une icône soit miraculeuse s'inscrit tout à fait dans la tradition orthodoxe : il n'est pas étonnant que le pope aime à raconter cette histoire, qui, par la démonstration directe de la grâce divine, légitime les choix théologiques d'une communauté marginale à bien des égards. Marion Bowman (2006), qui étudie les différents mouvements religieux et New Age à Glastonbury depuis plus de dix ans, fait référence à John Ives et à ce miracle que le pope " extrêmement excité » lui raconta en 1998. Elle parle aussi de l'aubépine que le pope avait plantée dans le jardin intérieur de sa boutique (mais il déménagea de High Street peu de temps après notre visite). En 2001, John Ives participa à la cérémonie annuelle de Noël (effectuée depuis 1929) autour de l'aubépine devant l'église anglicane, en présence du maire et des autres représentants des Églises chrétiennes (ce qui était une nouveauté, dans un effort pour promulguer le dialogue œcuménique) ; chaque année, le but de la cérémonie est de couper un rameau fleuri de l'aubépine et de l'envoyer à la Reine d'Angleterre. En 2005, John Ives ne participa pas à la cérémonie, mais un rameau fut conservé à son intention. Cela montre que Ives, qui représente l'EOC à Glastonbury, est bien intégré dans la société locale et qu'il est reconnu comme appartenant à une Église canonique ${ }^{11}$ par les autres «spécialistes du sacré ». D'ailleurs, à aucun moment M. Bowman ne se pose la question de savoir si l'EOC est une Église considérée comme canonique par d'autres Églises, orthodoxes en particulier, cela étant une question qui relève des relations entre Églises et non de la recherche scientifique.

Revenons sur cette icône de la Vierge, dont la composition est dominée par saint Joseph d'Arimathie à droite et saint Patrick à gauche. En effet, la particularité celtique de Glastonbury est soulignée par la présence de saints irlandais qui auraient marqué son histoire tels que saint Patrick, saint Benignus (disciple de saint Patrick), saint Indract, sainte Brigid et saint Columba. Parmi eux, saint Patrick est indéniablement la figure centrale : il aurait été le premier abbé de Glastonbury et enterré sur place ${ }^{12}$. C'est la réputation de Patrick qui semble avoir attiré les autres saints. Ainsi, poussé par le désir de 
visiter la tombe de ce dernier, saint Indract aurait décidé de s'arrêter dans cette "Seconde Rome ", "ainsi que s'appelait Glastonbury à l'époque à cause du nombre de saints qui y étaient enterrés » (Lewis, 1985 : 19). Il aurait été ensuite assassiné par des natifs tout près de Glastonbury. Saint David, patron du Pays de Galles, est aussi représenté. Ce dernier aurait été incité par un ange à se rendre à Jérusalem; le Patriarche l'aurait accueilli chaleureusement et lui aurait offert un autel portable, qu'il aurait ensuite ramené à Glastonbury. Ce lien avec un Patriarcat oriental prend évidemment toute son importance dans le cadre d'une réappropriation orthodoxe de ces saints celtiques.

14 Afin de créer une "mythologie sacrée » locale, on évoque donc la venue de personnages bibliques et de grands saints celtiques qui entretiennent entre eux des relations familiales ou spirituelles (de type "maitre-disciple »). Parallèlement, certains saints importants qui n'ont pas visité Glastonbury de leur vivant sont amenés à le faire après leur mort: plusieurs récits rapportent la translation de reliques via Glastonbury (Lewis, 1985 : 50-53), nouvel acte qui renforce la sacralité de l'endroit. Cette construction de "généalogies sacrées » et leur ancrage en ce lieu crée le sentiment d'une « concentration » continuelle et exceptionnelle de sainteté.

15 Étant donné l'histoire de Glastonbury et son rapport avec les origines du christianisme en Angleterre, il n'est pas étonnant qu'une "paroisse » orthodoxe revendiquant des racines celtiques se soit implantée à cet endroit. Pour les «Celtes orthodoxes" qui essaient de montrer que leur Église britannique puise directement aux sources du christianisme, sans la médiation de Rome, cet endroit devient central dans leur argumentation. Cette paroisse fait partie de l'EOC et, plus précisément, de l'éparchie ${ }^{13}$ de Grande-Bretagne, qui est basée à York. Il s'agit d'un autre endroit emblématique, dans la mesure où Constantin le Grand ${ }^{14}$ fut proclamé empereur par son armée à Eboracum (York) en 306.

Le 8 août 1999, la consécration épiscopale du père Stephen, responsable de l'éparchie de Grande-Bretagne, fit de lui « le gardien du Graal». Le sermon prononcé à cette occasion fait encore référence à Joseph d'Arimathie: «Saint Joseph a amené avec lui quelques solides compagnons, la Coupe de la Sainte Cène, ainsi que les reliques de sainte Anne, grand-mère du Christ, qui devint patronne de la Bretagne $»^{15}$. La tradition concernant Joseph d'Arimathie est ici remaniée afin d'intégrer sainte Anne, personnage qui est à la fois lié à l'Histoire Sainte et à une région " celtique ». Mais le thème celtique du Graal doit être mis en relation avec la religion orthodoxe à laquelle il est pourtant tout à fait étranger. Ainsi, Mgr Marc (2000:6) constate que «de nos jours, la quête du Graal intéresse toujours l'Orient chrétien ». Pour le prouver, il se réfère à une étude du père Boulgakov sur le sujet et au Patriarche de Constantinople, Athénagoras (1882-1972), qui « aimait se servir du symbole du Graal dans ses réflexions ».

La valorisation de hauts lieux, tels que Glastonbury, est accompagnée d'une autre démarche, celle de la restructuration du temps historique. Dans cette réinterprétation de l'histoire européenne, quatre grands événements sont évoqués afin d'expliquer la séparation progressive entre l'Église celtique et l'Église orthodoxe : le synode de Whitby en $664^{16}$; le Concile que Charlemagne organisa en 794 à Francfort-sur-le-Main et son couronnement consécutif par le Pape Léon $\mathrm{III}^{17}$; le schisme de $1054^{18}$; et enfin, la conquête normande ${ }^{19}$. La définition d'un christianisme celtique présuppose, en effet, deux opérations complémentaires : minimiser l'influence romaine et rechercher des liens avec l'Orient. 


\section{De l'histoire celtique à l'orthodoxie}

18 Sur le site de "L'Église Orthodoxe Celtique, éparchie de Suisse », figure une "Confession de foi ", selon laquelle les dévots veulent " par respect pour nos très illustres et héroïques pères celtes, selon la chair et selon l'esprit, assumer l'héritage légitime de culture et de sainteté qu'ils nous ont légué [...] malgré les siècles de romanisation forcée ». On introduit ici la notion d'un christianisme "originel», dont une forme aurait été l'ancienne expression occidentale de l'orthodoxie qu'il faudrait maintenant redécouvrir. Renouer les liens avec une structure ecclésiastique avec laquelle on a perdu contact à cause de facteurs extérieurs, retrouver ses racines perdues devient alors une mission à accomplir, une dette à payer envers ses ancêtres. La notion de "lignée croyante » introduite par D. Hervieu-Léger (1993 : 119) est ici pertinente. Définie comme «[...1]'expression visible d'une filiation que le croyant, individuel ou collectif, revendique expressément et qui le fait membre d'une communauté spirituelle qui rassemble les croyants passés, présents et futurs», elle prend ici le sens d'une filiation biologique, et non plus uniquement symbolique.

19 Le fondateur revendiqué par l'EOC est Jules Ferrette (il est aussi réclamé par d'autres Églises, comme la British Orthodox Church). Ce dominicain quitta l'Église catholique pour s'établir à Damas dans une mission presbytérienne irlandaise. Il aurait été ensuite nommé "évêque indépendant », sous le nom de Julius, par le métropolite œcuménique de l'Église orthodoxe syrienne en $1866^{20}$ : «En choisissant l'île d'Iona ${ }^{21}$ comme siège épiscopal, Mgr Ferrette se plaçait directement dans la tradition spirituelle de l'Église celtique. [...] Le siège d'Iona qui ne fut jamais un siège épiscopal était, pour l'évêque Ferrette, le symbole d'une orthodoxie occidentale d'origine apostolique qui ne fut jamais mêlée aux divisions de l'Église » (père Marc, 1995 : 75).

$20 \mathrm{Au}$ cœur de ce renouveau initié par Jules Ferrette, on trouve une distinction entre caractère et filiation apostolique. Si l'EOC légitime son caractère apostolique par la venue de Joseph d'Arimathie en Angleterre, elle reconnaitt pourtant que la filiation directe s'est interrompue avec la perte d'autonomie des Églises celtiques et leur soumission à Rome. C'est pourquoi la filiation avec le Patriarcat syrien d'Antioche fut utilisée pour renouer avec un siège apostolique autre que Rome, tout en gardant son indépendance. Ainsi, le site "Église Orthodoxe Celtique, éparchie de Suisse» contient-il une page intitulée "Lignée des patriarches de l'Église syrienne d'Antioche jusqu'en 1866 », dans laquelle on trouve la liste des patriarches d'Antioche, depuis l'apôtre Pierre jusqu'à Mar Ignatius Pierre III ${ }^{22}$ (celui-là même qui aurait consacré Jules Ferrette en 1866). L'appropriation de cette lignée a permis à l'EOC d'acquérir une double profondeur historique : lier l'héritage occidental, en particulier celtique, qu'évoque la légende de Joseph d'Arimathie, avec la tradition orthodoxe et orientale représentée par le Patriarcat d'Antioche.

21 Le rattachement à une Église orthodoxe « traditionnelle » a une efficacité non seulement symbolique, mais aussi pratique. Le but est de se voir transmettre la consécration épiscopale de manière canonique, c'est-à-dire par l'imposition des mains d'un évêque censé reproduire le geste des premiers apôtres. Tel fut le cas pour l'EOC. Après des siècles de rupture, la continuité de la lignée épiscopale n'étant plus assurée, elle a dû avoir recours à une autre Église pour renouer le fil de la succession ${ }^{23}$. Une fois cela accompli, elle a pu revendiquer un statut d'autocéphalie ${ }^{24}$ puisqu'il est toujours précisé que J. Ferrette fut ordonné comme évêque indépendant. En effet, après avoir été élevé au rang 
d'évêque, J. Ferrette se rendit en Grande-Bretagne, où il consacra en 1874 le premier Patriarche britannique: celui-ci était non seulement un pasteur de l'Église anglicane, mais aussi un barde gallois. Lors de son ordination, il prit le nom éloquent de Mar Pelagius I ${ }^{25}$ (Raoult, 1997 : 119).

L'itinéraire confessionnel du père écossais John Ross, tel qu'il est suggéré dans son texte The Orthodox Family diffusé sur Internet, illustre bien la nature instable et hésitante de ces noyaux d'orthodoxie en Occident, formés par des convertis «indigènes ». Leur survie dépend largement de la légitimité qu'une Église orthodoxe «traditionnelle » accepte de leur offrir, ou d'alliances avec d'autres groupes similaires. Ainsi, dans sa préface, J. Ross explique qu'avant de se convertir à l'orthodoxie en 1989, il était pasteur de l'Église épiscopale d'Écosse. En 1993, l'Église catholique orthodoxe de France ${ }^{26}$ (au sein de laquelle il fut ordonné pope) fut reniée par l'Église roumaine qui l'avait prise jusque-là sous sa juridiction. Il se trouva alors «sans abri » (homeless), «non canonique » (uncanonical) et isolé. C'est à ce moment qu'il s'est rattaché à la British Orthodox Church. En 1994, J. Ross a contribué à instaurer le dialogue entre cette Église et le Patriarcat copte. Mais, après son rattachement au Patriarcat copte, la British Orthodox Church a commencé de perdre son identité : le processus de "copticisation » qui s'est mit en place a amené plusieurs clercs et laïcs (voulant garder les traditions du christianisme celtique) à l'abandonner. Ces personnes se sont alors trouvées temporairement "dans la nature " (in the wilderness), jusqu'au moment où Mgr Mael, le primat actuel de l'EOC, leur a proposé de le rejoindre. Le parcours du père John Ross l'a donc mené non seulement d'une Église à l'autre, mais aussi dans un domaine où les frontières entre ce qui est «canonique » et ce qui ne l'est pas peuvent changer à tout moment.

L'élaboration d'une structure ecclésiale qui se veut, en même temps, indigène (liée à l'histoire occidentale) et orthodoxe, suscite la méfiance et est envisagée avec réticence par les hiérarchies ecclésiastiques en place qui peuvent y voir une perte d'adeptes et donc d'importance. Ce qui est perçu par les adeptes comme un caractère réformateur est considéré par les Églises traditionnelles comme une source d'illégitimité. À l'intérieur de l'EOC, ce sentiment d'instabilité prévaut. Lors des discussions sur le statut des paroisses en 2002, s'est posé le problème des reliques : à la mort d'un prêtre possédant des reliques, il fallait organiser que celles-ci reviennent à l'Église et non à ses héritiers; il fallait également trouver une solution pour que les reliques restent au sein de l'EOC au cas où un prêtre déciderait d'intégrer une autre Église, ou de faire scission ${ }^{27}$.

Une position marginale est souvent revendiquée. Les membres de cette Église ont une certaine prédilection pour des références contestées, comme Pélage et les Églises préchalcédoniennes ${ }^{28}$. Selon un membre de la paroisse de Paris, les dévots parisiens ne possèdent pas de lieu de rencontre et se retrouvent uniquement pour la messe : "On a toujours vécu dans les cavernes", nous dit-il. À une autre occasion, il déclare en plaisantant que quand cette Église sera construite, il partira : «Une Église est quelque chose de toujours en construction ». Ces personnes se perçoivent souvent comme les fondateurs de leur Église, à l'image des premiers temps du christianisme. Ils cherchent à retrouver ce qu'était la première communauté de Jérusalem: "C'est là notre quête du Graal », nous dit-on. Ces associations, plus ou moins explicites, leur permettent d'assumer tant les réactions négatives à leur égard, qu'une mission à part.

Huit patriarches se sont succédés à la tête de ce renouveau contemporain des Églises celtiques de confession orthodoxe. Mais l'histoire du monastère breton de la Sainte- 
Présence à Saint-Dolay n'est pas liée à un patriarche, comme nous le verrons, mais à un ermite.

\section{La fondation d'un monastère}

La biographie de cet ermite, dont le nom civil était Jean-Pierre Danyel, diffère selon les sources : selon la biographie qui circule au sein de l'EOC (père Marc, 1996 : 23-26), il est né le 22 juin 1917; ses parents l'abandonnent et il est placé à l'Assistance publique avant d'être recueilli par ses grands-parents, ou sa tante, à l'âge de cinq ans. Prisonnier de guerre, il est libéré en 1945 dans un état de santé grave. C'est au cours de son internement qu'il aurait trouvé la foi, grâce à un pasteur évangéliste belge. Il se retire donc dans un monastère (catholique) : « Il est convaincu que Dieu l'appelle au sacerdoce, mais selon les canons romains, il ne peut pas être prêtre parce qu'il est né de parents inconnus ${ }^{29}$. À l'époque, c'était une règle canonique incontournable » (ibid. : 23). Il est finalement baptisé en mai 1949 dans l'Église orthodoxe (sans plus de précisions, on ne sait donc laquelle), puis, en mai de la même année, il reçoit la consécration monastique. Ordonné prêtre au sein du Patriarcat de Glastonbury en 1953, il se retire, en 1955, dans une cabane, au fond d'un marécage, sur les lieux de l'actuel monastère de la Sainte-Présence. Selon Catherine Corvec (1993: 474), cet endroit rappelle les marais de Glastonbury, «la Jérusalem d'Occident ». Le choix de ce lieu - un «paradis celtique » faisant le «trait d'union entre l'ancienne religion druidique et la légende arthurienne » - n'aurait pas été uniquement motivé par la similitude des deux paysages, mais aussi par la volonté « de rebretonniser cette partie de la Bretagne où l'on parle le français depuis le douzième siècle ${ }^{30}$ » (Corvec, $1993:$ 478).

Deux ans plus tard, en janvier 1957, l'ermite reçoit la consécration épiscopale ${ }^{31}$ et prend alors le nom de Tugdual, l'un des sept saints fondateurs de Bretagne. Il meurt le 11 août 1968, à l'âge de cinquante et un ans, pratiquement seul ; cependant, il aurait prophétisé à deux personnes que dix ans après sa mort, il y aurait sur les lieux de son ermitage un monastère pour continuer son œuvre. C'est le 4 octobre 1977, «la dixième année de sa mort, sans avoir eu connaissance de cette prophétie » (père Marc, 1995: 78), que trois moines viennent s'installer au même endroit. L'ermite-fondateur sera canonisé par l'EOC le 11 août 1996.

Le fait que l'effort pour faire émerger un passé celtique oublié passe par la médiation d'un ermite s'accorde avec l'idée selon laquelle les Églises celtiques doivent leur caractère distinct au développement du monachisme. Les « Celtes orthodoxes » ne manquent pas de rappeler qu'à la suite de Jules Ferrette, Tugdual est bien le second refondateur de l'Église celtique car: "[...] on ne peut concevoir l'Église celtique sans cette formidable imprégnation de la spiritualité monastique " (père Marc, 1995: 76). Le monachisme marquerait donc la différence entre un modèle continental et des pratiques celtiques, tout en rapprochant ces dernières de l'idéal oriental ${ }^{32}$.

De l'Égypte et la Syrie à la Russie et Byzance, les références orthodoxes sont diversifiées et concomitantes; et elles nourrissent tant les discours que les pratiques de l'EOC. En mars 2003, et pour la troisième année consécutive, les moines du monastère breton sont allés en Égypte pour leur retraite annuelle. Près du monastère de Saint-Antoine, ils ont retrouvé le manguier que Mgr Mael avait planté l'année précédente et qu'il a arrosé avec une bouteille d'eau du puits de Saint-Dolay (Sainte Présence, 154-155, avril-mai 2003, p. 10). Cette offrande symbolique d'eau bretonne ${ }^{33}$, destinée à fertiliser la terre désertique 
d'Égypte, prend un autre sens quand on sait que Tugdual faisait boire aux visiteurs qui venaient solliciter son intercession l'eau du puits de son ermitage, qu'il considérait comme miraculeuse (père Marc, 1996 : 24). Mgr Mael a également offert une relique de saint Tugdual à l'abbé du monastère de Saint-Antoine : « Ainsi, le lien est établi entre nos deux monastères grâce à la présence de notre fondateur dans ce lieu que tous les moines regardent comme le premier monastère de la chrétienté » (ibid. : 11). En fait, le destin du saint fondateur et du groupe sont étroitement liés, l'un valorisant l'autre selon les circonstances : l'ermite ne devient saint que grâce à la dévotion des membres de cette Église ; quant à l'Église, elle peut, d'une part, se prévaloir d'un saint dans ses rangs, et, d'autre part, manipuler ses reliques (et les substances que le saint a touchées ou consacrées) afin d'établir des relations avec d'autres Églises.

Saint Tugdual constitue la référence centrale qui soude cette communauté. Dans une lettre écrite à l'occasion de l'ouverture du rassemblement de l'Église, en août 2002, Mgr Mael (alors âgé de soixante-dix-neuf ans) se présente en tant que membre de cette Église depuis quarante ans, prêtre depuis vingt-huit ans, évêque depuis vingt-deux ans et primat depuis sept ans. Il fait aussi référence à un autre ancien de l'Église, le père Gildas, prêtre doyen depuis trente-cinq ans et le seul de l'assemblée à avoir connu personnellement saint Tugdual : «Je n'ai été qu'en correspondance avec lui et n'ai pas eu la chance de le connaître en personne. Cependant, c'est à moi qu'ont été adressées ses dernières lettres avant sa naissance au ciel » (Sainte Présence, 147, septembre 2002, p. 5). Une fois ce synode terminé, les participants se sont accordés pour dire que l'esprit de saint Tugdual était présent au cours de ces journées. Il ne faut pas pour autant croire que le saint fondateur n'est évoqué que lors de grands rassemblements ou de voyages à l'étranger. Dans son éditorial (Sainte Présence, 152, février 2003), Mgr Marc présente les sources sur lesquelles les membres de cette Église s'appuient pour reconstituer la spiritualité du christianisme celtique, parmi lesquelles se trouvent principalement les écrits et l'esprit « de saint Tugdual et de saint François d'Assise " ${ }^{34}$.

31 Comment cette Église est-elle perçue dans la région? Selon un informateur habitant Rennes, Jean-Pierre Danyel (qui allait devenir Tugdual) commença son parcours par le séminaire, d'où il fut expulsé, puis essaya sans plus de succès de devenir pasteur. Ce serait ces échecs successifs qui l'auraient finalement conduit à l'orthodoxie ${ }^{35}$. Une fois évêque, il aurait décidé d'ordonner comme prêtre orthodoxe toute personne qui resterait trois jours au monastère; on ne sait donc pas combien de prêtres Tugdual aurait ainsi pu ordonner $^{36}$. La question des conversions est ici maximalisée et ridiculisée : le fait de lui attribuer une série de conversions successives et superficielles, à travers toutes les confessions chrétiennes, permet de discréditer non seulement la sincérité de son choix, mais aussi le choix de l'orthodoxie présentée comme l'option la moins « sérieuse ».

À ce sujet, un fidèle de l'EOC, vivant près de Morlaix, nous a raconté que l'évêque de Vannes fit placardé dans toutes les églises du diocèse un avis interdisant aux fidèles d'assister aux services de cette Église. Cependant, le prêtre catholique d'une commune voisine vint assister à une de ses cérémonies; le père Marc lui aurait alors demandé s'il voulait officier avec lui : il aurait refusé, mais accepté de communier. Ce récit est intéressant parce qu'il véhicule, d'une part, le sentiment de persécution des membres de cette Église ; et, d'autre part, il souligne la différence entre une hiérarchie ecclésiastique hostile (en la personne de l'évêque de Vannes) et un bas clergé amical. Ce fidèle a également conscience que son Église rencontre des difficultés avec les Églises orthodoxes traditionnelles : il nous a parlé d'un ami avec qui il avait fait le pèlerinage à pied jusqu'à 
Compostelle dans les années 1980. Puis ils se sont perdus de vue. Pendant qu'il découvrait l'EOC, son ami faisait un pèlerinage en Terre Sainte à la suite duquel il s'est converti à l'orthodoxie, puis a été récemment ordonné prêtre dépendant du Patriarcat de Constantinople. Lors de leurs retrouvailles, l'ami s'est montré très critique vis-à-vis de l'EOC qu'il considère comme n'étant pas canonique, en conséquence de quoi il refuse de donner la communion à notre informateur. Sa conclusion est que si son ami avait découvert l'EOC avant son pèlerinage en Terre Sainte, il serait aujourd'hui à leurs côtés, "parce qu'il est breton, il enseigne dans une école Diwan et s'intéresse au passé celtique ».

Qui sont les membres de cette Église ? Ce jeune homme de Morlaix était un de ceux qui circulaient pieds-nus (pour « son confort personnel »...), avait les cheveux longs et une apparence assez baba-cool. Il s'est fait baptiser dans l'EOC, avec pour marraine sa sœur, une catholique croyante. Lors de notre rencontre il était lecteur (grade des ordres mineurs). Avec d'autres fidèles de Morlaix, ils construisaient une église «avec leurs mains » qui, une fois terminée, devrait être consacrée par les moines de Saint-Dolay. Pour lui, la référence celtique est aussi importante (si ce n'est plus) que la référence orthodoxe.

Mais ce n'est pas toujours le cas : une femme originaire de Tarbes, iconographe, venue dans cette Église par son amour pour la peinture d'icônes. On lui demande de "celtiser " l'art des icônes mais elle trouve cette tâche difficile parce qu'elle a appris l'iconographie selon les principes byzantins. C'est elle qui a notamment réalisé l'icône de saint Tugdual exposée dans l'église de Saint-Dolay. Elle nous explique que ses icônes sont plutôt de type copte, plus « naïves ", plus proches de l'art populaire que les icônes byzantines; elles sont aussi entourées d'entrelacs celtiques. Pour une femme de Vannes, qui fait partie de la paroisse de Saint-Dolay avec son second mari (ils se sont mariés dans l'EOC) et ses enfants ${ }^{37}$, l'orthodoxie est aussi plus importante que la référence celtique. Elle dit apprécier tout particulièrement la relation avec les saints que l'Église orthodoxe privilégie (elle est sous la protection de la Vierge, un de ses enfants sous celle de saint Joseph et l'autre sous celle de saint François). Une autre femme, la tête toujours couverte, déclare qu'elle était catholique et voulait le rester, mais qu'elle se sent «chez elle » à l'EOC. Ayant voyagé à Jérusalem et au Liban, elle dit apprécier la liturgie orientale. Elle avoue avoir failli devenir intégriste à la suite de Vatican II avant d'avoir compris le sens de cette réforme. Une autre femme, depuis vingt ans dans l'EOC, a quitté Paris pour s'installer dans une commune voisine afin de pouvoir assister tous les jours à la liturgie du monastère.

Pour la plupart d'entre eux, la participation à l'EOC est associée à leur envie de participer à des rituels religieux forts de sens et élaborés. Les membres de l'EOC ne viennent pas tous chercher la même chose : certains privilégient les racines celtiques, d'autres sont plus sensibles à l'Orthodoxie. Ce balancement entre celtisme et orthodoxie dépasse largement le cadre des préférences individuelles pour s'inscrire dans l'histoire et le développement de l'EOC, dont les relations avec les mouvements néo-druidiques semblent fluctuer avec le temps ${ }^{38}$. Cela explique, en partie, les avis divergents exprimés à leur égard.

Marhic et Kerlidou (1996: 28) rapportent que cette Église « célèbre un rite orthodoxe occidental mêlant croix celtique, tribann, triskèle ${ }^{39}$ et croix grecque... ». Adoptant une attitude ironique, ces auteurs n'hésitent pas à caractériser l'EOC de pseudo-chrétienne et néo-païenne (ibid. : 188). Catherine Corvec associe la naissance du monastère breton aux néo-nazis du journal $\mathrm{La}$ Bretagne réelle, athées et néo-païens et qui voulaient provoquer « une nouvelle prise de conscience du fait breton par la religiosité » (Corvec, 1993: 473 et 
$478)^{40}$. En ce qui concerne l'ermite de Saint-Dolay, elle rapporte qu'il «célèbre des liturgies tout nu et se coiffe d'un panier à pain ou d'une passoire en guise de mitre, pour provoquer les journalistes» (ibid. : 474). En revanche, Jean-François Mayer (qui se réfère, entre autres, au monastère breton) et Pierre Erny adoptent une attitude moins critique, en acceptant même que "comme "mythe mobilisateur", l'idéal d'un rite orthodoxe occidental ne manque pas de force " (Mayer, 1997 : 211). Ces différentes appréciations montrent bien que ce mouvement religieux est sujet de controverse, non seulement pour les Églises, mais aussi pour les « spécialistes ».

\section{Entre syncrétisme et traditionalisme}

Dans leur effort pour valider et imposer le passé qu'ils ont recomposé comme une alternative chrétienne, les «Celtes orthodoxes » font appel au mouvement œcuménique ${ }^{41}$, considérant que ce dernier contribue à l'ouverture d'un dialogue interreligieux. Sans doute cela permet-il aussi de légitimer certains des emprunts faits à d'autres Églises, tout en désamorçant certaines critiques que l'on pourrait leur adresser quant à la canonicité de l'EOC. Dans Sainte Présence (156, juin 2003, p. 10), les échanges œcuméniques entre les jeunes de cette Église et «nos frères protestants » sont décrits. Durant leur voyage en Angleterre, les jeunes Français visitèrent Glastonbury où ils assistèrent à une messe donnée par le père John Ives, mais aussi aux vêpres dans la cathédrale de confession anglicane de Salisbury et à un culte évangélique.

En même temps, les "Celtes Orthodoxes" évoquant la crise de la foi en Occident, critiquent les changements opérés par Vatican II et les « excès » du Protestantisme ${ }^{42}$. Si le traditionalisme est une qualité de l'orthodoxie que l'on ne cesse d'exalter, il est aussi son point faible qui rend difficile l'approbation de ces nouvelles tendances occidentales. Ainsi, John Ross (The Orthodox family) désapprouve le refus des Orthodoxes de l'Est de permettre l'utilisation des langues occidentales dans la liturgie, tout en justifiant cette attitude qu'il attribue à leur peur d'être " contaminés » par les hérésies que ces convertis occidentaux risqueraient d'amener de leurs Églises. En évoquant le problème de la perte d'identité, il critique les convertis britanniques qui adoptent un pseudo-accent russe (« comme si cela leur permettait de devenir plus Orthodoxes!») ou des prénoms grecs (comme «Pancratios Macpherson» ou "Xenia Brown»). Selon lui, les convertis occidentaux sont traités comme des " citoyens orthodoxes de seconde zone ", sauf s'ils se mettent à imiter "les manières russes, grecques ou d'une autre culture étrangère ». Ces critiques désignent bien les limites de «l'entreprise syncrétique », au-delà desquelles on risque la dilution de sa spécificité.

Le discours des «Celtes orthodoxes » oscille entre la nécessité de préserver la tradition intacte et le souhait que chaque peuple puisse développer sa propre expression religieuse. Des contradictions de ce genre définissent également l'image des Celtes qu'érudits et folkloristes ont forgée. On les considère à la fois comme extrêmement conservateurs visà-vis de leurs traditions et comme les meilleurs représentants de certains traits de la modernité (en les qualifiant d'écologistes, féministes et individualistes). Les populations contemporaines, qui revendiquent une identité celtique, sont censées avoir intégré et conservé, à travers les siècles, des éléments païens dans leur pratique chrétienne; elles se veulent détentrices de valeurs que l'Occident, dans son développement matérialiste, est en train de perdre. Ainsi que le disent D. Hervieu-Léger et G. Davie (1996:286), « [...] les mouvements de renouveau religieux sont donc des analyseurs de la modernité 
occidentale $[\ldots$ et ...] la protestation socioreligieuse implicite ou explicite dont ils sont porteurs constitue, en creux, un révélateur des tendances de la modernité elle-même ».

C'est à cause de la « décadence » progressive de la civilisation occidentale (par perte de sa "celticité ») que le rapprochement avec l'Orient devient nécessaire, même si cela est présenté comme un retour et non une innovation. De ce point de vue, les Celtes représentent à la fois la quintessence de l'Occident et l'opposé de ce que cet Occident est devenu. La référence aux Celtes permet de considérer ce qui est positif dans la modernité comme étant ancien, c'est-à-dire celtique. Cette inversion des registres du temps, où le présent est disqualifié et le passé présenté comme la seule source d'un futur souhaitable, constitue une façon de dire que "nous avons toujours été modernes", et que l'oubli de l'héritage celtique marquerait la fin d'un modernisme éclairé. Pour les «Celtes orthodoxes ", la solution aux problèmes posés par la modernité se trouve non à l'Est, mais aux sources mêmes de l'Occident, c'est-à-dire dans l'héritage celtique dont les affinités avec la spiritualité orientale doivent être réappropriées. Toutes ces contradictions sont maniées afin de transformer l'exotisme du christianisme oriental en une qualité autochtone.

Le mouvement des «Celtes orthodoxes » ne doit pas être réduit à une simple variante du renouveau de la spiritualité celtique. Il reflète aussi l'intérêt croissant que l'orthodoxie suscite dans les pays occidentaux. La tendance générale est, dans ce cas, de ne pas reproduire simplement le modèle oriental, mais de l'adapter aux réalités locales. Pour les "Celtes orthodoxes", le détour par l'orthodoxie permet de nier les structures hégémoniques non seulement religieuses, telles que l'autorité romaine, mais aussi politiques, telles que la domination étatique anglaise ou française sur des régions "celtiques", afin de se ressourcer auprès d'un passé idéalisé. Ces associations ne prennent pas en compte certaines analyses présentant l'orthodoxie comme liée à l'exercice d'un pouvoir politique autoritaire et impérial (se référant à l'Empire byzantin et à la Russie).

42 Il existe, pourtant, un certain nombre de convertis occidentaux qui ne sont pas attirés par le Celtisme. Pour eux, le développement de ces nouveaux discours - quand ils restent modérés - constitue une occasion unique pour l'orthodoxie d'entrer dans la modernité occidentale, de quitter « le cocon oriental, byzantin et slave, qui fut pendant des siècles et son refuge et sa prison » (Behr-Sigel, 1998 : 37). L'Occident est ainsi présenté comme une source d'enrichissement pour l'orthodoxie.

Les «Celtes orthodoxes" essaient de réhabiliter ce qui est supposé être l'expression religieuse des populations celtiques avant qu'elles ne soient «opprimées». Cette tentative les oblige à imposer les Celtes comme les nouveaux médiateurs entre Occident et Orient et, par conséquent, comme un peuple lié à l'image utopique d'une Europe et d'une chrétienté harmonieuses. Redécouvrir cet âge d'or signifie explorer et faire revivre la période qui a précédé les ruptures au sein de l'Église et l'utilisation de la religion comme moyen de domination. Il suffit pourtant de poser le problème de la canonicité de ses nouvelles Églises pour que le poids de l'institution dissipe toute utopie romantique. 


\section{BIBLIOGRAPHIE}

AnsonPeter, 1964, Bishops at large, London, Faberand Faber.

Behr-Sigel Élisabeth, 1998, «L'ordination des femmes. Une question posée aussi aux Églises orthodoxes ", in Behr-Sigel Élisabeth, Ware Kallistos (Mgr), L'ordination des femmes dans l'Église orthodoxe, Paris, Éditions du Cerf, pp. 17-50.

BowmAn Marion, 2006, «The Holy Thorn Ceremony : revival, rivalry and civil religion in Glastonbury », Folklore, 117-2, pp. 123-140.

Corvec Catherine, 1996, « Une église pour les autonomistes : le cas de l'Église celtique », Ethnologie des faits religieux en Europe, Paris, CTHS, pp. 473-480.

CoXe Antony D., 1973, Haunted Britain. A guide to the Supernatural in England, Scotland and Wales, London, Pan.

DumVille David N., 1993, Saint Patrick. A.D. 493-1993, Woodbridge, The Boydell Press.

Erny Pierre, 1996, «Premiers pas d'une orthodoxie d'Occident », Ethnologie des faits religieux en Europe, Paris, CTHS, pp. 463-471.

ETCHINGHAM Colmán, 1999, Church Organisation in Ireland, A.D. 650 to 1000, Kildare, Laigin

Publications.

Hervieu-Léger Danièle, (avec la collaboration de Françoise Champion), 1987, Vers un nouveau christianisme? Introduction à la sociologie du christianisme occidental, Paris, Éditions du Cerf.

-, 1993, La religion pour mémoire, Paris, Éditions du Cerf, 1993.

Hervieu-Léger Danièle, Davie Grace, 1996, « Le déferlement spirituel des nouveaux mouvements religieux », in Davie Grace, Hervieu-Léger Danièle, Identités religieuses en Europe, Paris, Éditions de la Découverte, pp. 269-289.

LEWIS Lionel Smithett, 1985, Glastonbury, « The mother of Saints ». Her Saints. A.D. 37-1539, Orpington, Research Into Lost Knowledge Organisation, (1 ${ }^{\text {re }}$ éd. 1925).

Marc (Père), «L'Église Orthodoxe Celtique aujourd'hui », in Le christianisme celtique hier et aujourd'hui, Actes du colloque du 12 au 14 août 1995 organisé par le monastère de la Sainte-Présence, Saint-Dolay, pp. 73-82.

-, « La spiritualité de Mgr Tugdual, fondateur de l'ermitage de la Sainte-Présence », in Le christianisme celtique hier et aujourd'hui, Actes du colloque du 10 au 12 août 1996 organisé par le monastère de la Sainte-Présence, Saint-Dolay, pp. 23-38.

Marc (Mgr), 2000, Sources de la fondation apostolique de l'Église Celtique, Saint-Dolay.

Marhic Renaud, Kerlidou Alain, 1996, Sectes et mouvements initiatiques en Bretagne. (Du celtisme au nouvel âge), Rennes, Terre de Brume Éditions.

Mayer Jean-François, 1997, « L'Orthodoxie doit-elle être byzantine ? Les tentatives de création d'un rite oriental », in Ivanoff-Trinadtzaty Germain, Regards sur l'Orthodoxie. Mélanges offerts à Jacques Goudet, Lausanne, L'âge d'homme, pp. 191-213. 
Morvan Françoise, 2002, Le monde comme si. Nationalisme et dérive identitaire en Bretagne, Arles, Actes Sud.

Raoult Michel, 1997, Les druides. Les sociétés initiatiques celtiques contemporaines, Monaco, Éditions du Rocher.

Ross John (père), «L'Église Orthodoxe Celtique et les influences orientales », in Le christianisme celtique hier et aujourd'hui, Actes du colloque du 7 au 9 août 1999 organisé par le monastère de la SaintePrésence, Saint-Dolay, pp. 13-18.

Sims-Williams Patrick, 1998, "Celtomania and Celtoscepticism », Cambrian Medieval Celtic Studies, 36, p. 1-35.

Willaime Jean-Paul, 2004, Europe et religions. Les enjeux du XXI ${ }^{e}$ siècle, Paris, Fayard.

\section{NOTES}

1. Actes des colloques organisés par l'EOC et à la revue périodique qu'elle édite mensuellement, Sainte Présence. Notre enquête de terrain s'est déroulée en août 2002, quand les membres de l'Église se sont rassemblés à Saint-Dolay (Morbihan) pour discuter du statut des paroisses françaises.

2. Ce monastère ne figure évidemment pas dans le Petit guide des monastères orthodoxes de France, édité en novembre 2005 par le monastère de Cantauque, qui donne des informations sur les monastères canoniques, c'est-à-dire reconnus par les Églises orthodoxes traditionnelles. Encore faut-il ajouter que l'EOC n'est pas seule sur le créneau de la rencontre entre Orthodoxie et celtisme; il existe d'autres Églises revendiquant ces deux héritages et les combinant à leur manière propre.

3. Dans ce document, "l'Église Orthodoxe Celtique est déclarée en France sous le nom d'Association cultuelle de l'Église celtique apostolique ». Pour le fonctionnement de l'association, une cotisation annuelle minimum est fixée pour chaque membre. Lors des discussions pour l'établissement de ce règlement, les laïcs voulaient rendre la cotisation obligatoire, tandis que les clercs, plus souples, expliquaient: "c'est autre chose l'association, autre chose l'Église, on ne radie pas quelqu'un de l'Église parce qu'il n'a pas payé sa cotisation ». Chacune des huit paroisses francophones dispose d'une grande autonomie et d'un conseil paroissial; les délégués de paroisses siègent au conseil de leur éparchie (voir note 13) avec leur évêque.

4. À l'origine, la colline de Glastonbury était entourée d'un marécage, d'où son assimilation à une île.

5. Il s'agit du deuxième évêque français de l'EOC, le premier étant Mgr Mael, qui en est aussi le Primat. Dans notre texte, il est présenté tantôt comme le «père Marc » (avant sa consécration épiscopale) tantôt comme «Mgr Marc ». Au monastère de Saint-Dolay résidaient, au moment de notre enquête, cinq moines, un novice, et ces deux évêques.

6. Le fait d'associer un personnage de l'Histoire sainte avec la conversion d'une population locale est un topos qu'on retrouve, par exemple, dans la légende de la venue de Marie-Madeleine et de Lazare en Provence. Mgr Marc (2000 : 17) évoque même une tradition selon laquelle saint Joseph d'Arimathie serait « le jeune frère de Joachim et, par conséquent, l'oncle de la Vierge Marie ».

7. En Angleterre, un autre lieu est associé à une légende similaire : Priddy (Somerset). On raconte que le Christ y aurait passé une partie de ses « années perdues » (entre douze et trente ans); il y serait venu avec Joseph d'Arimathie qui visitait les mines d'étain de Cornouailles (Coxe, 1973 : 43). À l'inverse, afin de diminuer l'importance de Rome, on racontait que saint Pierre n'y était jamais allé et que la ville fut, en fait, convertie par des marchands juifs (Corvec, 1993 : 475). 
8. En Angleterre, les clercs protestants sont payés par l'État, et sont considérés, en quelque sorte, comme des fonctionnaires. Mais quand ils se convertissent et abandonnent leur charge, ils perdent du même coup leur source de revenu.

9. Il fait sans doute référence à saint Yves, saint breton du xiii ${ }^{\mathrm{e}}$ siècle, dont le nom est encore très répandu en Bretagne.

10. Selon les orthodoxes, il existe une réelle dichotomie entre le monde latin et le monde hellénistique, le premier ayant été évangélisé par saint Pierre et le second par saint Paul. Il est donc important pour les «Celtes Orthodoxes » de pouvoir se rattacher aux missions lancées par saint Paul et non saint Pierre.

11. En juin 2006, John Ives fut invité par la United Reformed Church de Glastonbury pour parler de la relation entre le christianisme et le New Age. Il se présenta comme un «archi-prêtre de l'EOC » dont la juridiction était certes très petite à Glastonbury, mais il rappela à son public que «l'Église Orthodoxe est la seconde plus grande Église au monde ». Ici encore, l'EOC est présentée comme une Église canonique. Notons également que quand Mgr Mael, accompagné de l'évêque Marc, a fait une tournée pastorale dans les îles Britanniques en automne 2003, il était à Bournemouth, où une paroisse de l'EOC est implantée (sous le nom de saint Joseph d'Arimathie), et il a été reçu par le maire et le chef de la police de Poole (Sainte Présence, novembre 2003, n. 161, p. 11). Il est clair qu'en Angleterre, cette Église est mieux intégrée et acceptée qu'en France, où plane sans cesse le soupçon d'avoir affaire à une secte (terme extrêmement péjoratif dans notre pays).

12. Selon la légende, il y a en fait deux Patrick: après sa mort, l'âme de saint Patrick d'Irlande alla rencontrer un autre saint Patrick, celui de Glastonbury (appelé Patrick l'Ancien et commémoré le 24 août), parce que les deux saints se seraient promis d'aller au ciel ensemble. Ainsi, du 18 mars jusqu'au 23 août, saint Patrick resta entouré par des anges en attendant le décès de Patrick l'Ancien. Pour une analyse très documentée de l'existence de ces deux saints Patrick à partir du ix e siècle, voir David Dumville (1993 : 59-64).

13. Dans les Églises orientales, cette subdivision territoriale correspond au diocèse de l'Église latine. Cependant, pour l'EOC, il s'agit de divisions nationales. On distingue cinq éparchies: France (primatiale), Grande-Bretagne, Amérique, Australie et Suisse. Sur le plan bureaucratique, chaque éparchie a son statut propre. Au moment de notre enquête, les éparchies de Suisse et d'Amérique n'avaient pas d'évêque, seulement des administrateurs. Selon Michel Raoult, qui se présente comme «docteur en maçonologie» (1997: 367), l'EOC aurait environ cinq cents membres actifs en France.

14. Cet empereur est reconnu comme un saint par l'Église orthodoxe et est fêté avec sa mère, sainte Hélène à qui l'on attribue la découverte de la croix du Christ. Il fonda Constantinople, capitale de l'Empire byzantin, lieu emblématique de sainteté dans le monde orthodoxe et siège actuel du Patriarcat.

15. Page «Homélie pour la consécration de Mgr Stephen», site «Église Orthodoxe Celtique, éparchie de Suisse ».

16. Il concernait le mode de calcul de la date de Pâques, le but étant de faire accepter le calcul romain. La réforme mit plusieurs dizaines d'années à être acceptée.

17. Ce concile a imposé la règle de saint Benoît dans les monastères et le modèle romain, tout en supprimant les usages liturgiques locaux.

18. On considère en fait que c'est le patriarcat romain qui « s'était écarté de l'unité de l'Église par la séparation de $1054 »$ (père John Ross, 1999 : 15).

19. Cette conquête se déroula en trois temps : en 1066, conquête de l'Angleterre; puis, plus progressivement, conquête du Pays de Galles ; enfin, en 1171, conquête de l'Irlande.

20. Il semblerait qu'il n'ait jamais pu présenter de preuve de cette ordination. De plus, en 1958, le patriarcat syriaque a nié toute validité aux ordinations de la succession Ferrette (Anson, 1964 : 250). Enfin, quand le «Patriarche de Glastonbury » (autrement dit le chef de la British Orthodox 
Church), dont dépendait l'EOC, a rejoint l'Église copte en 1994, celui-ci a été ordonné prêtre et consacré évêque (sous le nom de Seraphim de Glastonbury), car la succession apostolique dont se réclamait l'EOC n'était pas reconnue par les coptes.

21. Cette île au large de l'Écosse, dont le monastère fut fondé par saint Columba au vi ${ }^{e}$ siècle, est toujours considérée comme un haut lieu de la «spiritualité celtique ». Le Primat actuel de l'EOC porte encore le titre d' "évêque titulaire d'Iona ».

22. Le titre ecclésiastique de Mar est traditionnellement attribué aux évêques syriens, et signifie «Monseigneur ». Il sera utilisé par les successeurs de J. Ferrette.

23. Selon P. Erny (1993 : 468), la question du rattachement à une Église établie est importante, car il faut au moins deux évêques consécrateurs pour sacrer un nouvel évêque, selon le droit canonique orthodoxe. Et il faut trois évêques pour former un synode autonome.

24. Danièle Hervieu-Léger (1987: 146-148) rappelle que les communautés charismatiques du Renouveau essaient de maintenir des liens avec l'épiscopat et d'obtenir de la hiérarchie un statut canonique, tout en défendant leur droit à l'autorégulation.

25. Pélage, que l'on suppose d'origine britannique, prêcha au $v^{\mathrm{e}}$ siècle pour la puissance du libre arbitre, doctrine jugée hérétique à laquelle s'opposa saint Augustin.

26. Comme l'EOC, cette Église est accusée de recruter dans des « milieux se disant ésotéristes ou occultistes» (Erny, 1993: 470). L'auteur soutient que les influences de cette Église sont trop « orientales» (grecques ou russes) et son caractère autochtone reste encore éthéré (ibid. : 471), même s'il est très admiratif de la vieille liturgie des Gaules qu'elle a restaurée.

27. Nous nous trouvons en présence de communautés dont les frontières, autant que les membres, sont extrêmement fluctuants. En 1994, il y avait eu scission entre l'EOC et la British Orthodox Church quand cette dernière a décidé de se rattacher à la Coptic Orthodox Church. De même, en 1998, soit trois ans après sa consécration par Mgr Mael, Mgr Victrice (évêque de l'éparchie de Suisse) décida de quitter cette Église pour rejoindre l'Église copte française.

28. Ce qui unit ces Églises est leur rejet de la définition christologique du concile de Chalcédoine (451) : elles ne reconnaissent que les trois premiers conciles, Nicée (325), Constantinople (380) et Éphèse (431). Aujourd'hui, certains théologiens orthodoxes soutiennent que cette première fracture au sein de l'Église n'était due qu'à des différences de formulation.

29. On relèvera ici une petite incohérence : comment peut-on être «né de parents inconnus ", et avoir été recueilli par ses grands-parents ou sa tante?

30. Linguistiquement, la Bretagne est divisée en deux parties : dès le xii ${ }^{\mathrm{e}}$ siècle, on ne parle plus breton qu'à l'ouest d'une verticale passant par Saint-Malo et Dinan. Mais à cette division géographique, il faut ajouter une division sociale, puisque dès le xiie siècle aussi, les classes dirigeantes parlent français.

31. Consécration effectuée par trois évêques appartenant à de "petites » Églises orthodoxes : Mgr Irénée (Charles Borromée d'Eschevannes), primat de la Sainte Église catholique gallicane autocéphale, assisté de Mgr Julien Erni, évêque de Bienne du Siège Ecclésial Ecuménique, et de Mgr Eugène de Batchinsky, évêque titulaire de Berne et Genève de la Sainte Église orthodoxe ukrainienne conciliaire autocéphale en exil.

32. Le caractère monastique et supposé non hiérarchique (en ce sens plus proche du stéréotype orthodoxe que catholique) des Églises celtiques a été longtemps accrédité par la recherche. Ce n'est que depuis quelques années que la pertinence de ce modèle est remise en cause, voir C. Etchingham (1999).

33. Une association humanitaire "Présence » a été crée en 1997, et bien qu'il n'y soit fait nulle part mention de l'EOC, elle en est bien évidemment une émanation (mais cela n'a rien d'exceptionnel puisque chaque Église, grande ou petite, génère ce type d'associations). Cette association semble plus particulièrement active au Caire, en Égypte, où elle participe à l'aménagement d'un hôpital. On ne peut s'empêcher de voir là un autre moyen d'établir et de renforcer les liens entre les membres de l'EOC et cette «source d'orthodoxie " qu'est l'Égypte. 
Par ailleurs, la dimension caritative ne peut que crédibiliser l'Église elle-même. Plus généralement, les sociologues des religions ont montré que, dans un monde largement sécularisé, les actions caritatives des Églises restent un de leurs derniers points d'ancrage légitimes au sein de la sphère publique.

34. À l'instar de la construction de " généalogies sacrées » pour les saints de Glastonbury, on peut observer une opération parallèle, celle de la "celtisation» des grands saints européens postérieurs au Schisme (en conséquence de quoi ils ne sont pas vénérés par les Églises orthodoxes traditionnelles). C'est le cas pour saint François d'Assise (auquel on trouve d'innombrables références tant dans les textes que dans l'histoire de l'EOC), sainte Hildegarde de Bingen ou saint Nicolas de Flüe (auxquels sont consacrées deux contributions dans les actes des colloques organisés au monastère de la Sainte-Présence en 1995 et 1997).

35. Ce qui est présenté ici comme une succession d'échecs, trouve une tout autre connotation dans l'« hagiographie» des "Celtes Orthodoxes»: "Il se plonge alors dans l'étude des trois théologies: protestante, catholique et orthodoxe» (père Marc, 1995 : 76). D'autre part, nous avons vu que son "échec» au séminaire était présenté comme le résultat d'une "règle canonique » au sujet des enfants nés de parents inconnus et non d'un échec personnel.

36. Pour sa part, Anson (1964: 314-315) estimait les effectifs du métropolitat de Dol, mené par "Sa Blancheur Tugdual I ${ }^{\mathrm{er}}$ ", à dix évêques et deux ou trois laïcs.

37. Dont un garçon de 19 ans qui vient camper ici et part en vacances avec d'autres jeunes de la paroisse. Cette année-là, ils préparaient leur voyage en Irlande. Ces voyages retracent à chaque fois le parcours d'un saint, c'est ainsi qu'ils ont refait celui de saint Colomban en Europe. Ils partent pratiquement sans argent, et font des spectacles ou jouent de la musique dans la rue pour subvenir à leurs besoins. Ils sont souvent accueillis par le clergé d'autres Églises et célèbrent la messe dans des lieux emblématiques (comme la chapelle située au sommet du Croagh Patrick en Irlande) après en avoir demandé l'autorisation. Leurs aventures sont relatées dans la revue Sainte Présence, dans des textes portant des titres comme "Entre les mains de la Providence " (novembre 2003, n. 161, p. 7). Par ailleurs, nous avons rencontré à Saint-Dolay un autre jeune homme, lui aussi pied-nus en permanence et qui avait fait, à pied, le pèlerinage de Compostelle et celui de Jérusalem (qui a duré plus d'un an).

38. Pour donner un exemple précis de ce balancement, en 1967, Mar Georgius I (patriarche de Glastonbury et sixième Patriarche britannique) consacra Iltud, de son vrai nom Michel Raoult (1997), métropolite de l'Église celtique de Bretagne (ancêtre de l'EOC). Ce dernier conféra une « initiation spéciale » à trois moines démissionnaires de cette Église celtique pour fonder l'Ordre monastique d'Avallon (qui se voulait non chrétien). Puis, en 1974, deux anciens de cet Ordre fondèrent la Fraternité des Druides d'Occident. Iltud quitta cette Église en 1979, deux ans après la fondation du monastère de Saint-Dolay. Tout laisse supposer que c'est à ce moment qu'un premier mouvement vers l'orthodoxie se met en place, qui correspond aussi à une diminution de l'influence druidique sur cette Église.

39. Le tribann est un symbole représentant trois lignes verticales légèrement resserrées vers le haut, couramment utilisé et arboré par les néo-druides. Quant au triskèle, il est constitué de trois branches spiralées. Les deux sont associés à toutes sortes de triades symboliques.

40. On retrouve dans un grand nombre de publications des mouvements nationalistes bretons l'idée d'une opposition fondamentale entre "l'âme celtique» et la latinité. Dans ces circonstances, l'alliance entre panceltisme et pangermanisme se fait dès les années 1920 (Morvan, $2002: 173,200,215-216$ notamment).

41. Les "Celtes Orthodoxes" se présentent comme un mouvement d'envergure européenne, voire occidentale. Leur tendance à privilégier le dialogue œcuménique vérifie l'hypothèse d'un certain nombre de sociologues des religions qui soulignent l'articulation de l'entreprise œcuménique et de la construction de l'identité européenne. Comme le dit Jean-Paul Willaime (2004: 76) : «la construction de l'Europe est œcuménogène [...] mais elle engendre aussi des 
processus de reconfessionnalisation ». Ce qui est vrai aussi pour les "Celtes orthodoxes » qui recrutent essentiellement parmi les protestants et les catholiques.

42. Il peut sembler surprenant que des orthodoxes "réformistes " (au sens où ils souhaitent adapter l'orthodoxie à des convertis occidentaux et, dans ce cas précis, à une religiosité « celtique ») critiquent les tendances réformistes des catholiques et des protestants. Mais, il faut se souvenir qu'ils présentent leur mouvement comme un retour aux sources, et non comme une innovation.

\section{RÉSUMÉS}

Cet article analyse la rencontre opérée entre celtisme et orthodoxie, et le mouvement religieux qui en résulte, sous deux aspects : d'une part, les discours et, d'autre part, l'élaboration d'une structure ecclésiale. La venue de Joseph d'Arimathie en Grande-Bretagne constitue un épisode central dans les discours des «Celtes orthodoxes». Car la définition de ce christianisme celtique présuppose la construction d'une nouvelle «mythologie chrétienne » et la réinterprétation de l'histoire européenne. Le but est de minimiser l'influence romaine, tout en recherchant des liens avec l'Orient et les débuts du christianisme. En fait, c'est à cause de la "décadence " progressive de la civilisation occidentale, par perte de sa "celticité» (qui n'est pas perçue comme contradictoire avec le christianisme) que le rapprochement avec l'Orient est devenu nécessaire, même si cela est présenté comme un retour aux sources et non une innovation. En ce qui concerne l'élaboration d'une structure ecclésiale qui se veut, en même temps, indigène et orthodoxe, elle constitue une nouveauté, perçue par ses adeptes comme réformatrice mais critiquée par les Églises traditionnelles.

This article analyzes the encounter between Celticism and Orthodoxy, and the religious movement, which resulted from it, from two angles: on the one hand, the discourses, and on the other hand, the foundation of an ecclesiastical structure. The coming of Joseph of Arimathea in Great Britain constitutes a central episode in the discourses of "Celtic Orthodox". For the definition of this Celtic Christianity presupposes the construction of a new "Christian mythology" and the reinterpretation of the European history. The aim is to minimize the Roman influence, while searching for links with the Orient and the beginning of Christianity. It is because of the progressive "decay" of the occidental civilization, after having lost its "celticity" (which is not perceived as contradictory with Christianity) that the bringing together with the Orient seems to be necessary, even if it is presented to be a return, and not an innovation. As for the construction of an ecclesiastic structure, which wants to be, at the same time, indigenous and orthodox, it constitutes a novelty, perceived by its adepts as a reformation but criticized by traditional Churches.

Este artículo analiza el reencuentro que se ha producido entre Celtismo y Ortodoxia, y el movimiento religioso que resulta de este proceso, bajo dos aspectos : por un lado, los discursos, y por otro la elaboración de una estructura eclesial. La llegada de José de Arimatea a Gran Bretaña constituye un episodio central en los discursos de los "Celtas Ortodoxos". En efecto, la definición de este cristianismo celta presupone la construcción de una nueva "mitología cristiana" y la reinterpretación de la historia europea. El objetivo es minimizar la influencia romana, buscando los lazos con Oriente y los orígenes del cristianismo. De hecho, es a causa de la "decadencia" 
progresiva de la civilización occidental a causa de la pérdida de su "celticidad" (hecho que no es percibido como contradictorio con el cristianismo) que el acercamiento con Oriente se ha vuelto necesario, aunque éste sea presentado como un retorno a las fuentes y no como una innovación. La elaboración de una estructura eclesial que se quiere, al mismo tiempo, indígena y ortodoxa, por su parte, constituye una novedad, percibida por sus adeptos como reformadora pero criticada por las Iglesias tradicionales. (Trad. de Véronica Béliveau-Giménez)

\section{INDEX}

Keywords : Celtic Christianism, conversion, Europe, Orthodoxy, Religious renewal

Mots-clés : christianisme celtique, conversion, Europe, Orthodoxie, renouveau religieux

\section{AUTEURS}

\section{ALEXIS LÉONARD}

Centre d'Anthropologie de Toulouse / a.leonard@infonie.fr , Ross John (Father), 1999, « The Orthodox Family », 3e éd. rév., Pentecôte 1999, site " The British Eparchy of the Celtic Orthodox Church », http://www.abbess.demon.co.uk/, Site "Église Orthodoxe Celtique, éparchie de Suisse » : http://www.orthodox.ch/index.htm 